Variation in biochemical pathways

Several of the gene variants associated with metabolite levels, including those in or near the FADS cluster, ACADS, ACADM, ELOVL2 and $S L C 22 A 4$, had been previously reported ${ }^{10,12-14}$. As in other GWA studies, each associated variant implicates only a locus, and not necessarily a particular gene at that locus-although in this study, the variants often highlighted obvious candidate genes. In many instances, the associated metabolites are closely related to the activity of an enzyme encoded by a nearby gene (as in the case of ACADS and acylcarnitines), and sometimes the metabolites are a few steps removed from the known enzymatic activity (as with ELOVL2 and diacyl phosphatidylcholines). In other cases, the associated variants have no nearby genes with known roles in metabolism, suggesting that there may be previously unsuspected biochemical or physiological roles for genes at these loci. Of course, the effects of those genes on metabolite levels could be quite indirect.

Several of the identified loci are associated with metabolites that are risk factors for diseases, such as glucose levels or lipid levels ${ }^{4-7}$. Interestingly, some of the loci also have variants associated with risk of common diseases not thought to be directly influenced by metabolite levels: for example, SLC22A4 and Crohn's disease. However, the SLC22A4 variant reported in this paper as most strongly associated with carnitine levels is only partially correlated $\left(r^{2} \approx 0.4\right)$ with the variant most strongly associated with Crohn's disease ${ }^{15}$, so the overlap does not prove a causal connection between carnitine metabolism and the disease.

Illig et al. ${ }^{3}$ was limited to the analysis of genetic variants with a high minor allele fre- quency (here, $>10 \%$ ), typical for current GWA studies. Along these lines, it is notable that eight of the loci include four candidate genes (ACADS, $A C A D M, A C A D L$ and ETFDH) that have also been found to be mutated in rare, inborn errors of mitochondrial fatty acid oxidation. Such disorders are characterized by alterations in plasma metabolites such as glucose, ketone bodies and fatty acid intermediates. As Garrod first postulated, this suggests that the biochemical variation seen in the inborn errors of metabolism represent an extreme end on a continuum. Common variation at the same loci can give rise to more subtle biochemical phenotypes, and variants in the middle of this spectrum could have more significant consequences and yet fall short of causing a recognized inborn error of metabolism.

\section{Human biochemistry and disease}

Many biomarkers, including metabolite levels, show correlations with disease, but such correlation does not imply causality. Genetic variants that strongly influence biomarker levels can be tested for association in Mendelian randomization experiments (Fig. 1). In such experiments, there are two key elements: a biomarker that is correlated with disease, and a strong association between the biomarker and variation in a gene that is intimately tied to it (such as the protein encoding an enzyme directly involved in the biomarker's metabolism). If the variant shows the expected association with disease, this provides a strong argument in favor of causality, whereas the lack of an association in adequately powered samples that suggests the biomarker is correlated but not causal for disease. If multiple genetic loci associated with disease influence causal metabo- lites in a single pathway, enzymes in that pathway may emerge as tractable drug targets, and such results may also suggest mechanism-based markers for predicting disease and monitoring its progression.

Most exciting about the current work is the use of two global methodologies - genetic and metabolite profiling - to understand human in vivo metabolism. In the future, more comprehensive versions of the profiling technologies could be coupled to perturbations (for example, dietary challenges, drug treatments, aging) or used in combination with isotopic tracers to more directly infer the influence of genetic variation on in vivo reaction biochemistry and homeostasis. Just as Garrod's study of inborn errors of metabolism helped write a generation of textbooks on human biochemistry, so, potentially, could comprehensive studies of inborn variation of metabolism inform the next generation.

1. Garrod, A.E. Lancet ii, 1616-1620 (1902).

2. Garrod, A.E. Inborn Factors in Disease (Oxford University Press, Oxford, 1931).

3. IIlig, T. et al. Nat. Genet. 42, 137-141 (2010).

4. Kathiresan, S. et al. Nat. Genet. 41, 56-65 (2009).

5. Mohlke, K.L., Boehnke, M. \& Abecasis, G.R. Hum. Mol Genet. 17, R102-R108 (2008).

6. Dupuis, J. et al. Nat. Genet. 42, 105-116 (2010).

7. Saxena, R. Nat. Genet. 42, 142-148 (2010).

8. Keurentjes, J.J. et al. Nat. Genet. 38, 842-849 (2006).

9. Ferrara, C.T. et al. PLoS Genet. 4, e1000034 (2008).

10. Gieger, C. et al. PLoS Genet. 4, e1000282 (2008).

11. Skol, A.D., Scott, L.J., Abecasis, G.R. \& Boehnke, M. Nat. Genet. 38, 209-213 (2006).

12. Nagan, N. et al. Mol. Genet. Metab. 78, 239-246 (2003).

13. Peltekova, V.D. et al. Nat. Genet. 36, 471-475 (2004).

14. Tanaka, T. et al. PLoS Genet. 5, e1000338 (2009).

15. Silverberg, M.S. et al. Eur. J. Hum. Genet. 15, 328335 (2007)

\title{
Channelopathies converge on TRPV4
}

\author{
Bernd Nilius \& Grzegorz Owsianik
}

\section{Scapuloperoneal spinal muscular atrophy and Charcot-Marie-Tooth disease type $2 \mathrm{C}$ are inherited neurodegenerative diseases characterized by sensory defects and muscle weakness. Three new studies demonstrate that they are allelic disorders caused by mutations in the vanilloid transient receptor potential cation-channel gene TRPV4.}

Normal communication between the central nervous system and skeletal muscles requires intact efferent and afferent neuronal connections between spinal cord and muscle tissue.

Bernd Nilius and Grzegorz Owsianik are at the Department of Molecular Cell Biology, Katholieke Universiteit Leuven, Leuven, Belgium.

e-mail:bernd.nilius@med.kuleuven.be
Functionally, these connections depend on ion channels that maintain action potential propagation, synaptic transmission, plasticity and cell survival. Scapuloperoneal spinal muscle atrophy (SPSMA) and Charcot-MarieTooth disease type 2C (CMT2C, or hereditary motor sensory neuropathy type 2, HMSN IIC) are genetically heterogeneous inherited disorders caused by degeneration of peripheral nerves. Individuals with SPSMA show loss and progressive weakness of scapular and peroneal muscle tissue, bone abnormalities and laryngeal palsy. CMT2C, the most common inherited neurological disease, leads to progressive weakness of distal limbs, vocal cords, diaphragm, and intercostal and laryngeal muscles. Sensory deficits often impair hearing and vision. Bladder urgency and incontinence as well as bone abnormalities, such as scoliosis, are also common. 
SPSMA and CMT2C share clinical features, and the loci for both diseases map to chromosome 12q24.1-q24.31, suggesting that they might be clinical variants caused by defects in the same gene. Three studies in this issue ${ }^{1-3}$ now demonstrate that SPSMA and CMT2C are allelic disorders caused by mutations in TRPV4, which encodes a transient receptor potential (TRP) cation channel of the vanilloid subfamily.

\section{Versatile channel}

TRP cation channels are polymodal cellular sensors that integrate diverse physical and chemical stimuli in many physiological processes $^{4,5}$. TRPV4 is activated by innocuous heat, mechanical stress and extracellular hypotonicity. Potent agonists of TRPV4 include endogenous compounds such as anandamide, arachidonic acid and its cytochrome P450 metabolites, and synthetic phorbol esters.

Structurally, TRPV4 consists of six transmembrane segments (S1-S6), with a putative cation-permeating pore between S5 and S6. S3 and S4 seem to constitute an agonistbinding pocket that contributes to channel gating. The $\mathrm{N}$ and $\mathrm{C}$ termini face the cytoplasm and contain multiple domains ${ }^{6}$. As with other TRPV channels, the $\mathrm{N}$ terminus of TRPV4 contains six ankyrin (ANK) repeats linked by extraordinarily long finger-like folds ${ }^{7}$ (Fig. 1). A proline-rich domain (PRD) involved in mechanosensitivity is located proximal to the first ANK repeat. The central residues of the PRD, Pro132 and Pro144, interact with PACSIN3, a protein implicated in vesicular membrane trafficking, endocytosis and cytoskeleton reorganization ${ }^{8}$. The C-terminal domains, which include a TRP box and a calmodulin-binding site, are probably not relevant in this context.

The expression pattern of TRPV4 is relatively broad ${ }^{5}$ and only partially matches the disease symptoms typical of SPSMA and CMT2C. In the brain, TRPV4 is expressed in neurons of the vascular organ of the lamina terminalis, in anterior hypothalamic structures, and in ependymal cells of the choroid plexus in the lateral ventricles. It has also been found in motor, spinal ventral root and dorsal root ganglia neurons. Other prominent sites of TRPV4 expression include cartilage, bladder urothelium, kidney, vascular endothelium, pulmonary aortic smooth muscle and the inner ear ${ }^{4}$.

\section{TRPV4-related channelopathies}

By definition, channelopathies are diseases caused by mutations in ion channel genes, resulting in altered channel function.

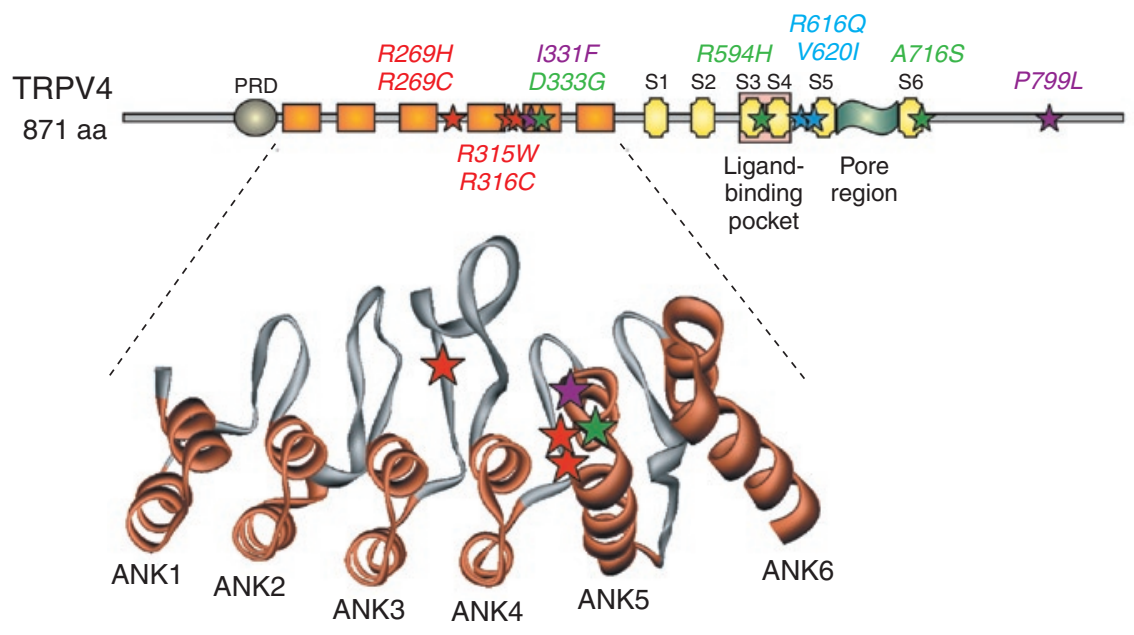

Spondylometaphyseal dysplasia, Kozlowski-type: D333G, R594H, A716S

Metatropic dysplasia: I331F, P799L

Brachyolmia: R616Q, V620I

Scapuloperoneal spinal muscle atrophy: $R 269 H, R 315 W, R 316 C$

Charcot-Marie-Tooth disease type 2C: R269C, R269H, R315W, R316C

Figure 1 TRPV4 mutations resulting in channelopathies. Predicted topology of human TRPV4 protein showing ankyrin repeats (ANK1-ANK6), a proline-rich domain (PRD), transmembrane segments (S1-S6), a ligand-binding pocket and a putative pore region. Red stars indicate mutations found in TRPV4-related scapuloperoneal spinal muscular atrophy and Charcot-Marie-Tooth disease.

Considering ion channels as highly specialized cellular devices, it is not surprising that changes to their structure might substantially alter their functionality. The first genetic analyses of TRPV4-related channelopathiesbrachyolmia, spondylometaphyseal dysplasia (SMD) and metatropic dysplasia-linked TRPV4 mutations to defects in bone development ${ }^{9,10}$. In contrast, the three new studies identify TRPV4 mutations as a pathogenic trigger in peripheral neuropathies ${ }^{1-3}$.

Using linkage analysis in families affected with SPSMA or CMT2C followed by candidate gene sequencing, Deng et al. ${ }^{2}$ identified two heterozygous TRPV4 mutations in two unrelated families. These mutations (C946CT and G806A) result in the amino acid substitutions R316C and R269H, respectively. In another study, Auer-Grumbach et al. ${ }^{1}$ identified these same two mutations, as well as a third heterozygous mutation (C946T) causing a R315W substitution, in five unrelated families with SPSMA or CMT2C. In a third study, Landouré et al. ${ }^{3}$ identified heterozygous TRPV4 mutations (C805CT and G806GA) causing the substitutions R269C and R269H in two families with CMT2C.

All the mutations identified affect residues located in the ANK region of TRPV4. Arg269 is located at the base of the third long fingerlike structure between ANK3 and ANK4, whereas Arg315 and neighboring Arg316 are part of ANK5 (Fig. 1) ${ }^{7}$. Importantly, the ANK region is thought to interact with regulatory proteins or participate in the self-association of TRPV4 homotetramers. Arg269 might also be a part of a putative biarginine (RXR) retention-retrieval motif involved in protein trafficking and multimerization ${ }^{11}$. It is also possible that the clustering of these positive charges in the $\mathrm{N}$-terminal region has a role in sequestering and binding of phosphatidylinositol 4,5-bisphosphate, a known regulator of TRP channels ${ }^{12}$.

\section{Functional dilemmas}

All three studies highlight an important role for TRPV4 in the pathogenesis of neurodegenerative diseases. However, a crucial question remains: how does TRPV4 function in motor and sensory peripheral nerves? Here the findings of the studies diverge and several puzzles emerge.

To explore the functional consequences of the TRPV4 mutations, all three groups used typical TRPV4 activation protocols, consisting of the application of heat, osmotic stress or agonists (such as $4 \alpha$-phorbols or arachidonic acid). Auer-Grumbach et al. ${ }^{1}$ found that HeLa cells transfected with constructs carrying the newly identified TRPV4 mutations did not respond to any stimulation. Furthermore, the TRPV4 mutants accumulated exclusively in the cytoplasm, 
whereas wild-type TRPV4 channels preferentially localized to the plasma membrane. Surprisingly, these data do not match the findings of the two other groups, who convincingly showed gain-of-function activity of the TRPV4 mutants in either HEK293 cells or Xenopus laevis oocytes ${ }^{2,3}$. Assuming that these discrepancies are related to differences in the experimental protocols, it will be important to clarify which of these phenotypes accurately reflects the behavior of the mutant TRPV4 proteins within their normal physiological context.

In considering all known disease-related mutations in TRPV4 (Fig. 1), links between disease symptoms and affected organs are not obvious. The bone dysplasia-associated mutations are scattered throughout the TRPV4 gene ${ }^{9,10}$. Interestingly, two gain-of-function alterations seen in SMD, I331F and D333G, are localized in close proximity to $\mathrm{R} 315 \mathrm{~W}$ and R316C. Although scoliosis seems to be a common symptom in CMT2 $\mathrm{C}^{3}$, individuals with SMD do not show any obvious defects of motor neurons. Furthermore, about $90 \%$ of individuals with CMT2C suffer from overactive bladders ${ }^{3}$, and it has already been shown that elevated TRPV4 activity might result in severe bladder overactivity ${ }^{13}$; surprisingly, however, individuals with SMD have no bladder symptoms at all. Considering the importance of TRPV4 in pain perception ${ }^{14}$, one would also expect to find hyperalgesia or altered nociception in individuals with SMD or CMT2C, which is clearly not the case. TRPV4, being activated by innocuous heat, is considered to be a cellular thermosensor. Interestingly, most individuals with CMT2C report worsening of hand weakness in the cold ${ }^{3}$.

Given the expression of TRPV4 in the inner ear, it is also not surprising that $59 \%$ of individuals with CMT2C have bilateral sensorineural hearing $\operatorname{loss}^{3}$. However, problems with osmoregulation, pulmonary hypertension or endothelial dysfunction, which might be anticipated from the TRPV4 expression profile, have not been described in TRPV4-related diseases. Although these functional dilemmas remain unresolved, the new studies suggest that TRPV4 could constitute a promising target for treatment of neurodegenerative diseases. Future research will bring further insight into the role of TRPV4 dysfunction in the pathomechanisms of these clinically diverse neurological diseases.

1. Auer-Grumbach, M. et al. Nat. Genet. 42, 160-164 (2010).

2. Deng, H.-X. et al. Nat. Genet. 42, 165-169 (2010).

3. Landouré, G. et al. Nat. Genet. 42, 170-174 (2010).

4. Nilius, B., Owsianik, G., Voets, T. \& Peters, J.A. Physiol. Rev. 87, 165-217 (2007).

5. Everaerts, W., Nilius, B. \& Owsianik, G. Prog. Biophys. Mol. Biol. published online, 10.1016/j.pbiomolbio. 2009.10.002 (14 October 2009).

6. Owsianik, G., D'Hoedt, D., Voets, T. \& Nilius, B. Rev. Physiol. Biochem. Pharmacol. 156, 61-90 (2006).

7. Phelps, C.B., Huang, R.J., Lishko, P.V., Wang, R.R. \& Gaudet, R. Biochemistry 47, 2476-2484 (2008).

8. Cuajungco, M.P. et al. J. Biol. Chem. 281, 1875318762 (2006).

9. Rock, M.J. et al. Nat. Genet. 40, 999-1003 (2008).

10. Krakow, D. et al. Am. J. Hum. Genet. 84, 307-315 (2009).

11. Zerangue, N., Schwappach, B., Jan, Y.N. \& Jan, L.Y. Neuron 22, 537-548 (1999).

12. Nilius, B., Owsianik, G. \& Voets, T. EMBO J. 27, 2809 2816 (2008).

13. Thorneloe, K.S. et al. J. Pharmacol. Exp. Ther. $\mathbf{3 2 6}$ 432-442 (2008).

14. Alessandri-Haber, N., Dina, O.A., Joseph, E.K., Reichling, D. \& Levine, J.D. J. Neurosci. 26, 3864 3874 (2006).

\title{
Deregulation of H3K27 methylation in cancer
}

\author{
Eva Martinez-Garcia \& Jonathan D Licht
}

\begin{abstract}
A new study now reports recurrent somatic mutation of EZH2, a histone methyltransferase that modifies $\mathrm{H} 3 \mathrm{~K} 27$, in diffuse large B-cell lymphoma (DLBCL). There is now evidence for both increased and decreased activity of enzymes controlling H3K27 methylation in cancer, suggesting that a precise balance of this methylation is critical for normal cell growth.
\end{abstract}

Powerful high-throughput sequencing technologies are now facilitating the identification of somatic mutations in cancer. On page 181 of this issue, Marco Marra and colleagues ${ }^{1}$ report next-generation sequencing of genomic and tumor DNA from an individual with diffuse large B-cell lymphoma (DLBCL) and discovery of a somatic mutation of the EZH2 gene, located within an exon encoding the catalytic SET domain of the protein. This mutation was found in $22 \%$ DLBCL-derived samples and $\sim 10 \%$ of follicular lymphoma-derived samples but not in mantle cell or T-cell lymphoma. EZH2 is a polycomb-group protein that mediates repression of gene transcription through its

Eva Martinez-Garcia and Jonathan D. Licht are in the Division of Hematology-Oncology, Northwestern University Feinberg School of Medicine, Chicago, Illinois, USA. e-mail:j-licht@northwestern.edu histone 3 lysine 27 (H3K27) methyltransferase activity. This suggests a role for aberrant regulation of $\mathrm{H} 3 \mathrm{~K} 27$ methylation in cancer.

\section{Histone methylation and gene expression}

Post-translational modification of histone tails, including lysine methylation, alters the physical state of chromatin and modulates gene expression. Chromatin immunoprecipitation-deep sequencing (ChIP-seq) has revealed that chromatin modifications are regulated in a temporal manner during development and in a precise spatial architecture across the genome. Promoters of actively transcribed genes are marked by the presence of a trimethyl mark on histone 3 lysine 4 (H3K4me3), whereas the transcribed body of an active gene is characterized by methylation at lysine $36(\mathrm{H} 3 \mathrm{~K} 36 \mathrm{me} 3)^{2}$. By contrast, inactive genes show methylation at lysine 27 (H3K27me3), and permanently silenced genes frequently are characterized by methylation at lysine 9 (H3K9me3) and heavy methylation of promoter-region $\mathrm{CpG}$ sites $^{3}$. Pluripotent embryonic stem cells are characterized by a set of genes with both the H3K4me3 and H3K27me3 marks-poised to be either silenced or activated as differentiation proceeds ${ }^{4}$.

Transcriptional cofactors containing the SET domain generally perform histone methylation. Histone demethylation is mediated by the Jumonji-C enzymes, which remove tri-, di- or monomethyl marks, and LSD1 can remove di- and monomethyl modifications from $\mathrm{H} 3 \mathrm{~K} 4$ and $\mathrm{H} 3 \mathrm{~K}^{5}$. Specific regions within transcriptional regulators, including the Chromo, Tudor, WD40 and PHD domains, bind methylated histone tails. Collectively the various histone modifications help determine the factors brought to a particular gene and the probability of that gene being expressed. 